\section{Prostatakarzinom: Prävention mit ASS?}

\section{Acetylsalicylsäure senkt die krankheitsspezifische Mortalitätsrate beim lokalisierten Prostatakarzinom, und zwar am effektivsten bei Hochrisiko- Patienten. Das ist das Ergebnis einer Analyse mit fast 6.000 Männern.}

mmer deutlicher zeichnen sich aktuellen Forschungsprojekten zufolge Zusammenhänge zwischen Krebserkrankungen und dem Gerinnungssystem ab. So stellt sich die Frage, inwieweit Wirkstoffe, die in die Mechanismen der Blutgerinnung eingreifen, auch die Entwicklung und das Wachstum von Tumoren beeinflussen können. Schon in früheren Studien waren sowohl ein chemopräventiver Effekt als auch eine therapeutische Wirkung von Acetylsalicylsäure (ASS) im Zusammenhang mit dem Prostatakarzinom aufgefallen. In der aktuellen Studie wurden nun Daten der Cancer of the Prostate Strategic Urologic Research Endeavor Database von 5.955 Männern mit einem lokalisierten Adenokarzinom der Prostata ausgewertet. Die Männer hatten sich entweder einer radikalen Prostatektomie unterzogen oder eine Radiotherapie erhalten. 2.175 der Patienten standen zusätzlich unter Antikoagulation (Warfarin, Clopidogrel, Enoxaparin und/ oder ASS).

Nach zehn Jahren waren $3 \%$ der Männer, die Antikoagulanzien (AK) eingenommen hatten an ihrem Prostatakarzinom verstorben, aus der Kontrollgruppe dagegen $8 \%$. Auch Rezidive und Knochenmetastasen traten unter AK deutlich seltener auf ( $28 \%$ vs. $36 \%$ bzw. $3 \%$ vs. $6 \%)$. Um herauszufinden, welche Patienten besonders von Gerinnungshemmern profitieren, wurden Subgruppenanalysen herangezogen. Hier zeigte sich der größte Überlebensvorteil für Männer mit Hochrisiko-Karzinomen. Sie erreichten unter Antikoagulanzien eine krankheitsspezifische Zehn-Jahres-Mortalität von $4 \%$ gegenüber $19 \%$ in der entsprechenden Kontrollgruppe. Wie der Vergleich der verschiedenen Antikoagulanzien zeigte, war dies allerdings überwiegend dem ASS zuzuschreiben, das $84 \%$ der Männer dieser Gruppe einnahmen. Ob die Krebstherapie durch Prostatektomie oder Bestrahlung erfolgte, hatte dabei keinen Einfluss. Unter Berücksichtigung verschiedener Variablen sank die spezifische Mortalität unter ASS etwa um die Hälfte, während sich bei anderen $\mathrm{Ge}$ rinnungshemmern kein signifikanter Effekt ergab.

Fazit: Die Ergebnisse der Studie untermauern die Hypothese eines chemopräventiven und antineoplastischen Effekts von ASS. Daten, die hierzu schon für das Kolorektalkarzinom vorliegen scheinen sich auch für das Prostatakarzinom zu bestätigen. Bevor allerdings eine Empfehlung für eine Begleittherapie gegeben werden kann, müssen die optimale Dosierung sowie mögliche Risiken in einer prospektiven Studie untersucht werden.

Dr. Christine Starostzik

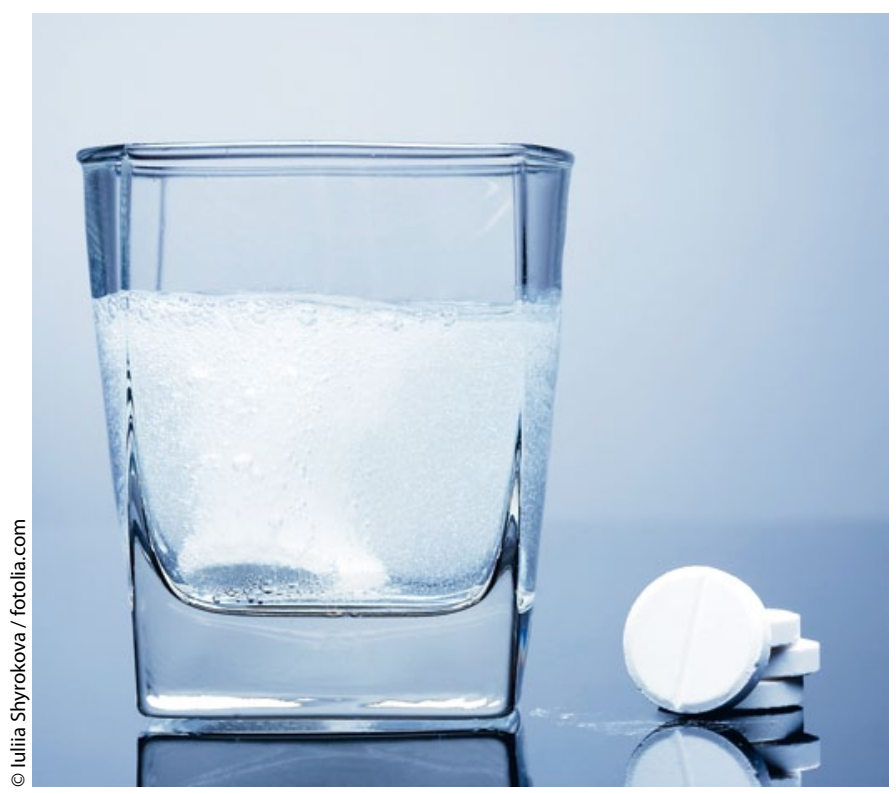

Der Einsatz von ASS reicht mittlerweile weit über den Kopfschmerz hinaus. Auch Krebspatienten sollen jetzt profitieren. 